\title{
Effectiveness of a Short Duration of Neoadjuvant Endocrine Therapy in Patients with HR+ Breast Cancer-An NCDB Analysis (2004-2016)
}

\author{
Macy M. Goldbach, BS ${ }^{1}$ (D), Laura Burkbauer, BA ${ }^{1}$, Tina Bharani, MD ${ }^{1}$, Austin D. Williams, MD, MSEd ${ }^{3}$, \\ Luke Keele, $\mathrm{PhD}^{2}$, Jami Rothman, $\mathrm{MD}^{1,4}$, Rachel Jankowitz, $\mathrm{MD}^{4}$, and Julia C. Tchou, MD, PhD, FACS ${ }^{1,4}$ \\ ${ }^{1}$ Division of Endocrine and Oncologic Surgery, Department of Surgery, Perelman School of Medicine, University of \\ Pennsylvania, Philadelphia, PA; ${ }^{2}$ Division of Epidemiology and Biostatistics, Department of Surgery, Perelman School of \\ Medicine, University of Pennsylvania, Philadelphia, PA; ${ }^{3}$ Department of Surgery, Lankenau Medical Center, Wynnewood, \\ PA; ${ }^{4}$ Rena Rowan Breast Center, Abramson Cancer Center, Perelman School of Medicine, University of Pennsylvania, \\ Philadelphia, PA
}

\begin{abstract}
Background. National medical/surgical organizations have recommended the use of neoadjuvant endocrine therapy (NET) to bridge surgery delay of weeks to months for patients with hormone receptor positive $(\mathrm{HR}+)$ breast cancer during the ongoing coronavirus disease 2019 (COVID-19) pandemic. The effects of NET of varying durations on pathologic response are unclear. Using the National Cancer Database (NCDB), we evaluated objective response to short ( $<9$ weeks), moderate (9-27 weeks), and long ( $>27$ weeks) duration of NET.

Patients and Methods. The study cohort included female patients diagnosed with nonmetastatic invasive HR+ breast cancer, stratifying by those who received NET versus no NET between 2004 and 2016. Pathologic
\end{abstract}

Macy M. Goldbach and Laura Burkbauer are co-first authors.

Supplementary Information The online version contains supplementary material available at https://doi.org/10.1245/s10434021-10287-5.

(C) Society of Surgical Oncology 2021, corrected publication 2021

First Received: 2 February 2021

Accepted: 28 May 2021;

Published Online: 14 June 2021

M. M. Goldbach, BS

e-mail: Julia.Tchou@pennmedicine.upenn.edu

J. C. Tchou, MD, PhD, FACS

e-mail: Julia.Tchou@pennmedicine.upenn.edu response was grouped into four categories (complete, downstaged, stable, upstaged) by comparing clinical and pathologic staging data. Objective response to NET included complete, downstaged, and stable pathologic response. Clinical characteristics were compared using $\chi^{2}$ and analysis of variance (ANOVA) tests. Multivariable logistic regression was used to determine factors associated with NET use and objective response according to NET duration.

Results. A minority (1.2\%) received NET in our cohort. Factors associated with NET use included older age, nonBlack patients, more advanced clinical stage, higher comorbidity score, government insurance, and lobular histology. Objective response rate (ORR) was $56.7 \%$, $52.1 \%$, and $49.0 \%$ after short, moderate, and long NET duration, respectively.

Conclusion. Short NET duration did not result in an inferior ORR. Future study to evaluate the interaction between surgery delay and NET use on clinical outcome will provide insights into the safety of NET to bridge potential surgery delay in patients with $\mathrm{HR}+$ breast cancer.

Hormone receptor positive (HR+) breast cancer, defined as breast cancer that expresses estrogen (ER) and/or progesterone receptors (PR) and lacks HER-2/neu (HER2) expression, is the most common subtype of breast cancer, accounting for over $70 \%$ of all breast cancer cases. ${ }^{1}$ Although endocrine therapy is widely used in the adjuvant setting, it is infrequently used in the neoadjuvant setting. Neoadjuvant endocrine therapy (NET) is usually 
recommended for those who will not tolerate neoadjuvant chemotherapy (NAC) in the USA. ${ }^{2}$ However, there has been a recent resurgence of interest in the use of NET in the setting of the coronavirus disease 2019 (COVID-19) pandemic. $^{3-9}$ National medical and surgical organizations, including the American Society of Breast Surgeons (ASBrS), the Commission on Cancer (CoC), and the Society of Surgical Oncology (SSO), have recommended the use of NET to bridge possible surgery delays during the pandemic to allow for conservation of limited resources such as personal protective equipment and ventilators. ${ }^{3-9}$ In addition, during the pandemic, patients who may otherwise require NAC to downstage their disease are likely to receive NET instead, with the goal of sparing these patients from the immunosuppressive side effects of chemotherapy that may increase the risk of COVID-19 infection in this population. $^{10}$

A recent meta-analysis has compared the efficacy of NET and NAC and reported similar pathologic response rates between the two neoadjuvant treatment strategies. Importantly, NET was associated with significantly lower adverse events and toxicity. ${ }^{10}$ Historically, the most common NET duration used in clinical trials and in the clinics has been reported to range from 12 to 24 weeks. ${ }^{10,11}$ In the context of the pandemic, the duration of NET is likely to vary, but it is anticipated to be shorter than the reported duration and will depend on both regional COVID-19 disease trends and when treatment facilities have the capacity to resume elective surgery. The impact of a shorter NET duration on disease outcomes such as objective response is, however, unclear. Furthermore, whether the use of NET may ameliorate the potential deleterious consequences of surgery delay is unknown. ${ }^{12}$

As an attempt to understand real-world experience of NET in the setting of its utility during the novel COVID-19 pandemic, we designed this study using data from the National Cancer Database (NCDB) to evaluate the impact of NET use on the objective response rate (ORR) as stratified by three NET durations, defined as short $(<9$ weeks), moderate (9-27 weeks), and long ( $>27$ weeks), in patients with $\mathrm{HR}+$ breast cancer. The primary outcome of this study was to determine the ORR according to short, moderate, or long NET duration, focusing on shorter NET duration, which may have been commonly employed during the COVID-19 pandemic. The secondary outcome of this study was to determine clinical factors associated with objective response to NET.

\section{MATERIALS AND METHODS}

\section{Cohort Selection}

The study cohort consisted of female patients diagnosed with nonmetastatic, invasive $\mathrm{HR}+$ breast cancer between 2004 and 2016 identified in the National Cancer Database (NCDB). The NCDB is a clinical oncology database that acquires data from more than 1500 Commission on Cancer-accredited facilities with estimated capture of $75 \%$ of all new cancer diagnoses. ${ }^{13}$ Institutional review board approval was not required for this study since data from the NCDB are deidentified. We identified 811,802 patients who met inclusion criteria (Fig. 1). Patients with unknown or no surgery $(n=37,121)$, unknown chemotherapy $(n=$ $18,139)$, or unknown endocrine therapy $(n=16,615)$ were excluded.

We defined patients who received preoperative therapy as those who started either chemotherapy or endocrine therapy $\geq 14$ days before surgery. Time elapsed between DX_CHEMO_STARTED_DAYS or DX_HORMONE_STARTED_DAYS and DX_SURG_STARTED_DAYS was used to determine whether patients received preoperative therapy or no preoperative therapy. Patients who received both neoadjuvant chemotherapy (NAC) and neoadjuvant endocrine therapy (NET) $(n=3427)$ or NAC only $(n=$ $29,735)$ were excluded. Our final study cohort consisted of

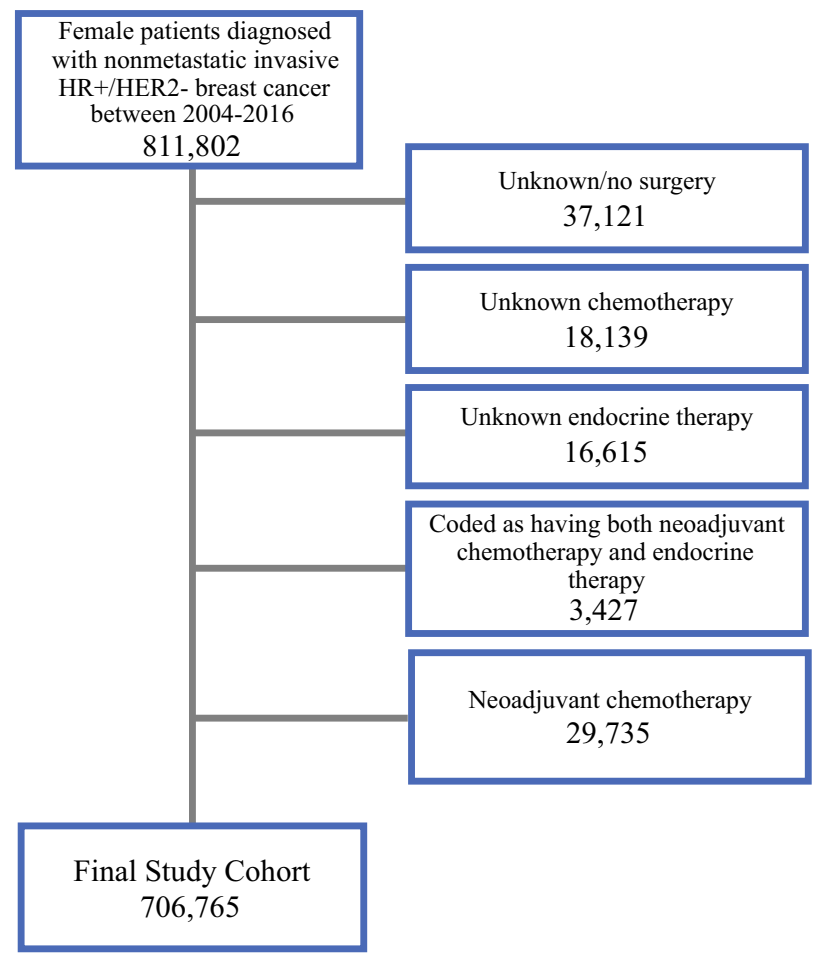

FIG. 1 Consort diagram depicting inclusion and exclusion criteria used to establish study cohort 
706,765 patients, of whom $698,439(98.8 \%)$ received no NET (surgery first) versus 8326 (1.2\%) received NET. Time elapsed between DX_HORMONE_STARTED_ DAYS and DX_SURG_STARTED_DAYS was also used as a proxy to determine the duration of NET. A histogram was generated to determine the frequency of NET durations in our cohort (Fig. 2). We then stratified NET duration into three intervals (short, moderate, and long) based on the first quartile ( $<9$ weeks), interquartile range (9-27 weeks), and fourth quartile ( $>27$ weeks).

\section{Definition of Objective Response to NET}

Pathologic response to NET was grouped into four categories (complete response, downstaged, stable, and upstaged disease) by comparing clinical and pathologic tumor and nodal staging data (Supplementary Table 1). Treatment response was considered to be complete when pathologic stage was stage 0 . Treatment response was considered "downstaged" when pathologic stage was lower than clinical stage. Treatment response was considered stable when pathologic stage remained the same as clinical stage. Treatment response was considered "upstaged" when pathologic stage was higher than clinical stage. Overall pathologic response was the summation of treatment response in both tumor (breast) and nodes (axilla) (Supplementary Table 2). We defined objective response in this study to include those with (1) complete pathologic response, (2) downstaged disease, and (3) stable disease after NET.

\section{Statistical Analysis}

Demographic and clinical characteristics were compared using $\chi^{2}$ tests for categorical variables and ANOVA tests for continuous variables. Multivariable logistic regression was performed to determine factors associated with NET use and factors associated with objective response to NET. Poisson regression was performed to evaluate trends in the use of NET over time. All hypothesis tests were two-sided, and $p<0.05$ was considered statistically significant. STATA 16/SE software was used to carry out all statistical analyses (StataCorp LLC, College Station, TX).

\section{RESULTS}

\section{Cohort Characteristics}

Clinical characteristics of our study cohort $(n=706,765)$ stratified by receipt of NET and no NET (surgery first) are summarized in Table 1 . Overall, only a minority $(n=8326$, or $1.2 \%$ ) received NET. The two groups differed in all clinical variables included in our analysis. Patients who received NET were noted to be older, had higher CharlsonDeyo (CD) comorbidity scores, had government insurance, were more likely to be treated at academic programs,
FIG. 2 Histogram of NET duration in weeks for our study cohort

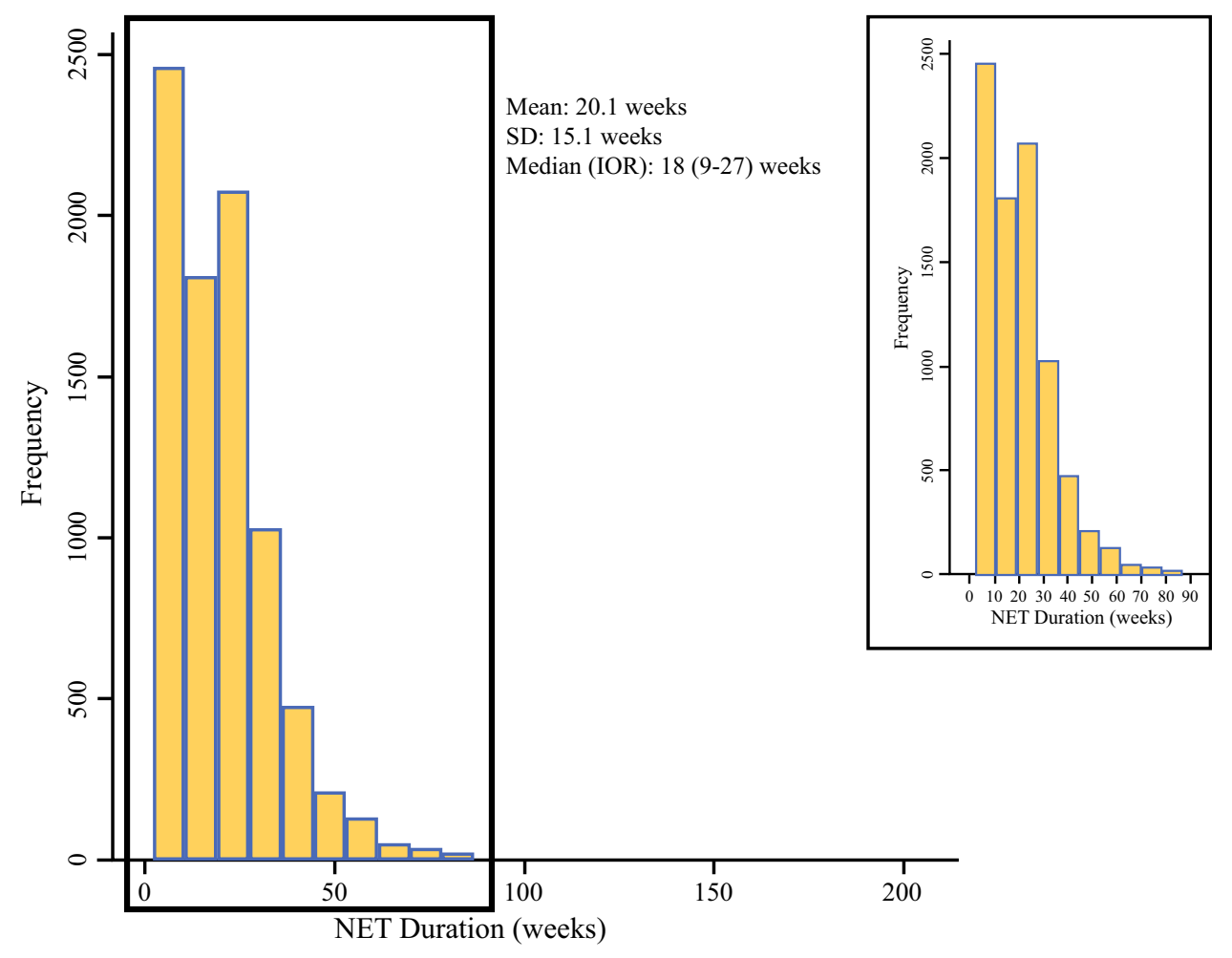


TABLE 1 Demographic and clinical characteristics of females diagnosed with nonmetastatic invasive HR+/HER2- breast cancer stratified by no preoperative therapy (surgery first) versus neoadjuvant endocrine therapy (NET)

\begin{tabular}{|c|c|c|c|c|c|c|c|}
\hline & \multicolumn{2}{|l|}{ Overall } & \multicolumn{2}{|c|}{ No NET (Surgery First) } & \multicolumn{2}{|l|}{ NET } & \multirow[t]{2}{*}{$P$} \\
\hline & $\mathrm{N}$ & $\%$ & $\mathrm{~N}$ & $\%$ & $\mathrm{~N}$ & $\%$ & \\
\hline$n=706,765$ & 706,765 & $100.0 \%$ & 698,439 & $98.8 \%$ & 8326 & $1.2 \%$ & \\
\hline Age at Diagnosis (mean $\pm(\mathrm{SD}))$ & \multicolumn{2}{|l|}{$62.8 \pm 12.6$} & \multicolumn{2}{|l|}{$62.7 \pm 12.6$} & \multicolumn{2}{|c|}{$67.7 \pm 12.1$} & $<0.001$ \\
\hline \multicolumn{8}{|l|}{ Year of Diagnosis } \\
\hline 2010-2013 & 382,364 & $54.1 \%$ & 378,255 & $54.2 \%$ & 4109 & $49.4 \%$ & \multirow[t]{2}{*}{$<0.001$} \\
\hline 2014-2016 & 324,401 & $45.9 \%$ & 320,184 & $45.8 \%$ & 4217 & $50.6 \%$ & \\
\hline \multicolumn{8}{|l|}{ Race } \\
\hline White & 607,750 & $86.0 \%$ & 600,642 & $86.0 \%$ & 7108 & $85.4 \%$ & \multirow[t]{4}{*}{0.03} \\
\hline Black & 62,112 & $8.8 \%$ & 61,310 & $8.8 \%$ & 802 & $9.6 \%$ & \\
\hline Asian/PI & 23,902 & $3.4 \%$ & 23,642 & $3.4 \%$ & 260 & $3.1 \%$ & \\
\hline Other/Unknown & 13,001 & $1.8 \%$ & 12,845 & $1.8 \%$ & 156 & $1.9 \%$ & \\
\hline \multicolumn{8}{|l|}{ Comorbidity Score } \\
\hline 0 & 581,580 & $82.3 \%$ & 575,003 & $82.3 \%$ & 6577 & $79.0 \%$ & \multirow[t]{4}{*}{$<0.001$} \\
\hline 1 & 98,212 & $13.9 \%$ & 96,963 & $13.9 \%$ & 1249 & $15.0 \%$ & \\
\hline 2 & 19,996 & $2.8 \%$ & 19,646 & $2.8 \%$ & 350 & $4.2 \%$ & \\
\hline $3+$ & 6977 & $1.0 \%$ & 6827 & $1.0 \%$ & 150 & $1.8 \%$ & \\
\hline \multicolumn{8}{|l|}{ Insurance Status } \\
\hline Private Insurance & 347,107 & $49.1 \%$ & 344,238 & $49.3 \%$ & 2869 & $34.5 \%$ & \multirow[t]{3}{*}{$<0.001$} \\
\hline Government & 342,047 & $48.4 \%$ & 336,909 & $48.2 \%$ & 5138 & $61.7 \%$ & \\
\hline Other/Unknown & 17,611 & $2.5 \%$ & 17,292 & $2.5 \%$ & 319 & $3.8 \%$ & \\
\hline \multicolumn{8}{|l|}{ Hospital Category } \\
\hline Community & 65,853 & $9.3 \%$ & 65,338 & $9.4 \%$ & 515 & $6.2 \%$ & \multirow[t]{5}{*}{$<0.001$} \\
\hline Comprehensive Community & 312,008 & $44.1 \%$ & 308,826 & $44.2 \%$ & 3182 & $38.2 \%$ & \\
\hline Academic & 208,288 & $29.5 \%$ & 205,169 & $29.4 \%$ & 3119 & $37.5 \%$ & \\
\hline Integrated Network Cancer Program & 100,302 & $14.2 \%$ & 98,881 & $14.2 \%$ & 1421 & $17.1 \%$ & \\
\hline Other/Unknown & 20,314 & $2.9 \%$ & 20,225 & $2.9 \%$ & 89 & $1.1 \%$ & \\
\hline \multicolumn{8}{|l|}{ Clinical Stage } \\
\hline \multicolumn{8}{|l|}{ Overall } \\
\hline 1 & 447,180 & $63.3 \%$ & 444,854 & $63.7 \%$ & 2326 & $27.9 \%$ & \multirow[t]{4}{*}{$<0.001$} \\
\hline 2 & 160,016 & $22.6 \%$ & 155,637 & $22.3 \%$ & 4379 & $52.6 \%$ & \\
\hline 3 & 19,647 & $2.8 \%$ & 18,432 & $2.6 \%$ & 1215 & $14.6 \%$ & \\
\hline Unknown & 79,922 & $11.3 \%$ & 79,516 & $11.4 \%$ & 406 & $4.9 \%$ & \\
\hline \multicolumn{8}{|l|}{ Tumor Stage } \\
\hline 1 & 474,223 & $67.1 \%$ & 471,644 & $67.5 \%$ & 2579 & $31.0 \%$ & $<0.001$ \\
\hline 2 & 140,017 & $19.8 \%$ & 136,358 & $19.5 \%$ & 3659 & $43.9 \%$ & \\
\hline 3 & 17,587 & $2.5 \%$ & 16,476 & $2.4 \%$ & 1111 & $13.3 \%$ & \\
\hline 4 & 5748 & $0.8 \%$ & 5036 & $0.7 \%$ & 712 & $8.6 \%$ & \\
\hline Unknown & 69,190 & $9.8 \%$ & 68,925 & $9.9 \%$ & 265 & $3.2 \%$ & \\
\hline Nodal Stage & & & & & & & \\
\hline 0 & 603,034 & $85.3 \%$ & 596,702 & $85.4 \%$ & 6332 & $76.1 \%$ & $<0.001$ \\
\hline 1 & 51,324 & $7.3 \%$ & 49,907 & $7.1 \%$ & 1417 & $17.0 \%$ & \\
\hline $2 / 3$ & 9230 & $1.3 \%$ & 8937 & $1.3 \%$ & 293 & $3.5 \%$ & \\
\hline Unknown & 43,177 & $6.1 \%$ & 42,893 & $6.1 \%$ & 284 & $3.4 \%$ & \\
\hline Histology & & & & & & & \\
\hline Ductal & 551,165 & $78.0 \%$ & 545,407 & $78.1 \%$ & 5758 & $69.2 \%$ & $<0.001$ \\
\hline Lobular & 87,263 & $12.3 \%$ & 85,539 & $12.2 \%$ & 1724 & $20.7 \%$ & \\
\hline Other/Unknown & 68,337 & $9.7 \%$ & 67,493 & $9.7 \%$ & 844 & $10.1 \%$ & \\
\hline
\end{tabular}


TABLE 1 continued

$\begin{array}{llllll}\text { Overall } & \frac{\text { No NET (Surgery First) }}{\mathrm{N}} & \frac{\mathrm{NET}}{\mathrm{N}} & \frac{\mathrm{N}}{\%} & P\end{array}$

\begin{tabular}{|c|c|c|c|c|c|c|c|}
\hline \multicolumn{8}{|l|}{ Tumor Grade } \\
\hline Well Differentiated & 214,714 & $30.4 \%$ & 212,305 & $30.4 \%$ & 2409 & $28.9 \%$ & \multirow[t]{4}{*}{$<0.001$} \\
\hline Moderately Differentiated & 344,871 & $48.8 \%$ & 340,503 & $48.8 \%$ & 4368 & $52.5 \%$ & \\
\hline Poorly/Undifferentiated & 113,664 & $16.1 \%$ & 112,627 & $16.1 \%$ & 1037 & $12.5 \%$ & \\
\hline Unknown & 33,516 & $4.7 \%$ & 33,004 & $4.7 \%$ & 512 & $6.1 \%$ & \\
\hline \multicolumn{8}{|l|}{ Surgery } \\
\hline \multicolumn{8}{|l|}{ Primary } \\
\hline Lumpectomy & 438,587 & $62.1 \%$ & 434,240 & $62.2 \%$ & 4347 & $52.2 \%$ & \multirow[t]{3}{*}{$<0.001$} \\
\hline Mastectomy & 267,642 & $37.9 \%$ & 263,669 & $37.8 \%$ & 3973 & $47.7 \%$ & \\
\hline Surgery, NOS & 536 & $0.1 \%$ & 530 & $0.1 \%$ & 6 & $0.1 \%$ & \\
\hline \multicolumn{8}{|l|}{ Axilla (*2012 and later) } \\
\hline None & 33,756 & $4.8 \%$ & 32,900 & $4.7 \%$ & 856 & $10.3 \%$ & \multirow[t]{5}{*}{$<0.001$} \\
\hline SLN & 348,296 & $49.3 \%$ & 344,917 & $49.4 \%$ & 3379 & $40.6 \%$ & \\
\hline ALND & 51,050 & $7.2 \%$ & 49,966 & $7.2 \%$ & 1084 & $13.0 \%$ & \\
\hline $\mathrm{SLN}+\mathrm{ALND}$ & 90,773 & $12.8 \%$ & 89,643 & $12.8 \%$ & 1130 & $13.6 \%$ & \\
\hline Other/Unknown & 182,890 & $25.9 \%$ & 181,013 & $25.9 \%$ & 1877 & $22.5 \%$ & \\
\hline \multicolumn{8}{|l|}{ Adjuvant Radiation } \\
\hline \multicolumn{8}{|l|}{ Post-Lumpectomy Radiation } \\
\hline Yes & 363,915 & $83.0 \%$ & 360,908 & $83.1 \%$ & 3007 & $69.2 \%$ & \multirow[t]{3}{*}{$<0.001$} \\
\hline None & 73,798 & $16.8 \%$ & 72,485 & $16.7 \%$ & 1313 & $30.2 \%$ & \\
\hline Unknown & 874 & $0.2 \%$ & 847 & $0.2 \%$ & 27 & $0.6 \%$ & \\
\hline \multicolumn{8}{|l|}{ Post-Mastectomy Radiation } \\
\hline Yes & 66,587 & $24.9 \%$ & 65,179 & $24.7 \%$ & 1408 & $35.4 \%$ & \multirow[t]{3}{*}{$<0.001$} \\
\hline No & 200,147 & $74.8 \%$ & 197,603 & $74.9 \%$ & 2544 & $64.0 \%$ & \\
\hline Unknown & 908 & $0.3 \%$ & 887 & $0.3 \%$ & 21 & $0.5 \%$ & \\
\hline Treatment Duration, median(IQR) (weeks) ${ }^{\mathrm{a}}$ & $18(9-27)$ & & NA & & $18(9$ & & NA \\
\hline \multicolumn{8}{|l|}{ Recurrence Score (Pre-TAILORX) } \\
\hline$<18$ & 122,562 & $59.3 \%$ & 121,549 & $59.3 \%$ & 1013 & $57.9 \%$ & \multirow[t]{3}{*}{$<0.001$} \\
\hline $18-30$ & 67,536 & $32.7 \%$ & 66,901 & $32.6 \%$ & 635 & $36.3 \%$ & \\
\hline$>30$ & 16,723 & $8.1 \%$ & 16,620 & $8.1 \%$ & 103 & $5.9 \%$ & \\
\hline Follow-up (median (IQR)) (months) ${ }^{\mathrm{b}}$ & $41(24-61)$ & & $41(24-61)$ & & $37(2$ & & $<0.001$ \\
\hline Total Deceased ${ }^{\mathrm{c}}$ & 51,157 & $7.2 \%$ & 50,011 & $7.2 \%$ & 1146 & $13.8 \%$ & $<0.001$ \\
\hline
\end{tabular}

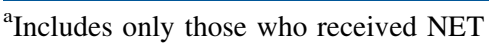

${ }^{\mathrm{b}}$ Follow-up represents the elapsed time between diagnosis and last contact

${ }^{\mathrm{c}}$ Vital status available for $(597,151,84.5 \%)$ patients in cohort

$N E T$ neoadjuvant endocrine therapy, $S D$ standard deviation, $P I$ Pacific Islander, $S L N$ sentinel lymph node biopsy, $A L N D$ axillary lymph node dissection, $I Q R$ interquartile range, NOS not otherwise specified, $N A$ not applicable

presented at higher clinical stage, had lobular histology, had higher tumor grade, and more often underwent mastectomy and axilla node dissection procedures. Unexpectedly, patients in the NET group were less likely to have a recurrence score $>30(p<0.001)$. Length of follow-up was shorter in the NET group (37 months versus 41 months, $p<0.001$ ). Despite shorter median follow-up time, patients in the NET group had a higher unadjusted death rate than the surgery-first group (13.8\% versus $7.2 \%$, $p<0.001)$.

\section{Clinical Characteristics Associated with NET Use}

Multivariable logistic regression analysis was performed to identify clinical factors associated with NET use. Results are presented in Table 2. Older age ( $\geq 50)$, more 
recent diagnosis (2014-2016), CD comorbidity score $\geq 2$, government insurance, treatment at a setting other than community hospital, higher clinical tumor and nodal stage, and lobular histology were significantly associated with NET use. Patients with clinical T4 (locally advanced) tumors were most likely to receive NET [odds ratio (OR) 25.54, $p<0.001]$. Of note, compared with their white counterparts, NET was less likely to be used in Black patients (OR 0.91, $p=0.01$ ).

Objective Response Associated with NET Duration

To examine the effect of NET duration on treatment response, we first evaluated if the varying NET duration in our cohort followed a bell-shaped curve distribution. As

TABLE 2 Multivariable logistic regression of factors associated with NET use

\begin{tabular}{|c|c|c|c|c|c|}
\hline & OR & $(95 \% \mathrm{CI})$ & & & $p$ \\
\hline \multicolumn{6}{|l|}{ Age at diagnosis } \\
\hline$<50$ & 1.00 & - & to & - & - \\
\hline $50+$ & 2.29 & 2.09 & to & 2.52 & $<0.001$ \\
\hline \multicolumn{6}{|l|}{ Year of diagnosis } \\
\hline 2010-2013 & 1.00 & - & to & - & - \\
\hline 2014-2016 & 1.25 & 1.20 & to & 1.31 & $<0.001$ \\
\hline \multicolumn{6}{|l|}{ Race } \\
\hline White & 1.00 & - & to & - & - \\
\hline Black & 0.91 & 0.84 & to & 0.98 & 0.01 \\
\hline Asian/PI & 0.97 & 0.85 & to & 1.11 & 0.65 \\
\hline Other/Unknown & 0.96 & 0.81 & to & 1.14 & 0.67 \\
\hline \multicolumn{6}{|l|}{ Charlson-Deyo comorbidity score } \\
\hline 0 & 1.00 & - & to & - & - \\
\hline 1 & 0.96 & 0.90 & to & 1.03 & 0.24 \\
\hline 2 & 1.21 & 1.08 & to & 1.36 & 0.001 \\
\hline $3+$ & 1.31 & 1.10 & to & 1.55 & 0.002 \\
\hline \multicolumn{6}{|l|}{ Insurance status } \\
\hline Private insurance & 1.00 & - & to & - & - \\
\hline Government & 1.58 & 1.51 & to & 1.67 & $<0.001$ \\
\hline Other/unknown & 1.91 & 1.69 & to & 2.17 & $<0.001$ \\
\hline \multicolumn{6}{|l|}{ Hospital category } \\
\hline Community & 1.00 & - & to & - & - \\
\hline Comprehensive community & 1.41 & 1.28 & to & 1.56 & $<0.001$ \\
\hline Academic & 2.22 & 2.01 & to & 2.45 & $<0.001$ \\
\hline Integrated network cancer program & 2.02 & 1.82 & to & 2.25 & $<0.001$ \\
\hline \multicolumn{6}{|l|}{ Clinical tumor stage } \\
\hline 1 & 1.00 & - & to & - & - \\
\hline 2 & 5.14 & 4.88 & to & 5.42 & $<0.001$ \\
\hline 3 & 12.68 & 11.71 & to & 13.73 & $<0.001$ \\
\hline 4 & 25.54 & 23.22 & to & 28.09 & $<0.001$ \\
\hline \multicolumn{6}{|l|}{ Clinical nodal stage } \\
\hline 0 & 1.00 & - & to & - & - \\
\hline 1 & 1.12 & 1.05 & to & 1.19 & 0.001 \\
\hline $2 / 3$ & 0.87 & 0.76 & to & 0.98 & 0.03 \\
\hline \multicolumn{6}{|l|}{ Histology } \\
\hline Ductal & 1.00 & - & to & - & - \\
\hline Lobular & 1.32 & 1.25 & to & 1.40 & $<0.001$ \\
\hline Other/unknown & 1.01 & 0.94 & to & 1.09 & 0.72 \\
\hline
\end{tabular}

Patients with available information for all variables included in multivariable model $(n=608,873)$ 
shown in the histogram depicted in Figure 2, a bimodal distribution was noted. The most common NET duration was $\leq 9$ weeks, followed by NET duration $\sim 24$ weeks. The mean NET duration was $20.1 \pm 15.1$ weeks. We then stratified NET duration into three intervals: short, moderate, and long or $<9,9-27$, and $>27$ weeks, respectively.

The primary outcome of this study was to determine the objective response to NET according to duration of use. Overall, the objective response rate (ORR) was $56.7 \%$, $52.1 \%$, and $49.0 \%$, while the proportion of patients with upstaged disease was $25.9 \%, 23.4 \%$, and $19.0 \%$, after short, moderate, and long NET duration, respectively (Table 3). Clinical characteristics of the three NET duration groups were compared (Table 3). As expected, patient characteristics differed significantly across the three groups, except for CD comorbidity score $(p=0.51)$, tumor grade $(p=0.50)$, and length of follow-up $(p=0.22)$. Patients who received moderate and long NET duration were more likely to present with higher clinical stage $(p<$ 0.001).

A secondary outcome of this study was to determine clinical factors associated with objective response to NET. A multivariable logistic regression analysis was performed, and results are depicted in a forest plot (Fig. 3). Objective response was similar between short and moderate (OR $1.00, p=0.90$ ) or long duration (OR 1.13, $p=0.17$ ) of NET use (Fig. 3). Patients who were older ( $\geq 50$ years; OR 1.42, $p<0.01$ ), of Asian/Pacific Islander descent (OR 1.44, $p=$ 0.04 ), or with clinical T4 (OR 1.72, $p<0.01$ ) or N2/3 disease (OR 3.34, $p<0.01$ ) were more likely to have an objective response to NET. Patients with a CD comorbidity score of 2 (but not 3) were less likely to have an objective response to NET (OR 0.64, $p<0.01$ ). Unexpectedly, tumors with lobular histology (OR 0.56, $p<0.01$ ) and higher tumor grade (poorly/undifferentiated, OR 0.59, $p<$ 0.01 ; moderately differentiated, OR $0.70, p<0.01$ ) were less likely to have an objective response to NET.

To further improve the applicability of our results for the COVID-19 pandemic, we performed a subset analysis of patients with clinical stage I and II disease $(n=6705)$ whose disease stage is probably similar to those recommended to receive NET during the pandemic. Clinical factors associated with NET use and factors associated with objective response to NET use are summarized in Supplementary Table 3 and Supplementary Figure 1. Results were similar to those reported above for the entire cohort. The ORR was $60.5 \%, 54.9 \%$, and $50.8 \%$, while the proportion of patients with upstaged disease was $28.3 \%$, $25.9 \%$, and $21.4 \%$, after short, moderate, and long NET duration, respectively. Objective response was similar between short and moderate (OR 1.00, $p=0.95)$ or long NET duration (OR 1.12, $p=0.22$ ).
Changes in NET Practice Pattern Trend between 2010 and 2016

NET was not commonly used in the USA during the study period. As described earlier, between 2010 and 2016, only $1.2 \%$ of patients received NET prior to surgery. However, we noted a small but significant rise in the use of NET from $1 \%$ in 2010 to $1.3 \%$ in 2016 [incidence rate ratio $($ IRR $)=1.10, p<0.001]$ (Supplementary Figure 2). The median NET duration lengthened from 16.0 weeks to 20.2 weeks between 2010 and 2016. We also observed a lengthening of mean NET duration from 19.2 weeks in 2010 to 20.2 weeks in $2016(p=0.01)$ (Supplementary Figure 3).

\section{DISCUSSION}

Although historically underutilized, NET is now gaining a foothold in the management of early-stage $\mathrm{HR}+$ breast cancers, propelled in part by an unprecedented need to delay surgeries, minimize clinic visits, and conserve limited resources in the wake of the current pandemic. $^{2,5}$ In this study, we report that the ORR was $56.7 \%, 52.1 \%$, and $49.0 \%$ after short, moderate, and long NET duration, respectively. A short NET duration did not result in an inferior objective response. Our findings were comparable to prior studies, albeit of much smaller sample size, which have reported ORR ranging from $20 \%$ to $76 \% .{ }^{14}$ We also showed a small proportion of patients, $25.9 \%, 23.4 \%$, and $19.0 \%$, with disease upstaged after short, moderate, and long NET duration, respectively. These results were also consistent with previous findings. ${ }^{8}$

Previous studies, including multiple prospective trials, have evaluated the effects of NET over periods of 12-24 weeks, with some extending as long as a year. ${ }^{10,11,14}$ Given the recommendations by major surgical and oncologic associations to use NET as a bridge to surgery during the ongoing pandemic, ${ }^{3-9}$ we anticipate shorter NET durations than those used in formal studies. Our analysis of the NCDB data shows that shorter durations of NET use are already in effect in real-world practice, with $25 \%$ of patients receiving $<9$ weeks of NET. Furthermore, ORR was actually highest in those patients receiving short duration of NET (56.7\%), highlighting the utility of NET as a bridge to surgery when surgical delays are expected. Objective response in this group continued to be high $(60.5 \%)$ when we restricted our analysis to patients with clinical stage I and II disease, who are most likely to receive NET during the pandemic. As disease upstaging can also occur over this time frame in a sizable minority of patients, we advise interval clinical exam $+/-$ imaging to determine which patients have poor response to NET and may require accelerated surgical intervention. 
TABLE 3 Demographic and clinical characteristics of patients who received NET stratified by duration of NET (weeks)

\begin{tabular}{|c|c|c|c|c|c|c|c|}
\hline & \multicolumn{6}{|c|}{ NET Duration } & \multirow[t]{3}{*}{$P$} \\
\hline & \multicolumn{2}{|l|}{$<9$ weeks } & \multicolumn{2}{|l|}{ 9-27 weeks } & \multicolumn{2}{|l|}{$>27$ weeks } & \\
\hline & $N$ & $\%$ & $N$ & $\%$ & $n$ & $\%$ & \\
\hline$n=8326$ & 2045 & $24.6 \%$ & 4294 & $51.6 \%$ & 1987 & $23.9 \%$ & \\
\hline Age at Diagnosis (mean $\pm(\mathrm{SD}))$ & $64.8 \pm 12.9$ & & $68.0 \pm 11.8$ & & $69.7 \pm 11.5$ & & $<0.001$ \\
\hline \multicolumn{8}{|l|}{ Year of Diagnosis } \\
\hline $2010-2013$ & 1101 & $53.8 \%$ & 2068 & $48.2 \%$ & 940 & $47.3 \%$ & \multirow[t]{2}{*}{$<0.001$} \\
\hline 2014-2016 & 944 & $46.2 \%$ & 2226 & $51.8 \%$ & 1047 & $52.7 \%$ & \\
\hline \multicolumn{8}{|l|}{ Race } \\
\hline White & 1750 & $85.6 \%$ & 3706 & $86.3 \%$ & 1652 & $83.1 \%$ & \multirow[t]{4}{*}{0.001} \\
\hline Black & 185 & $9.0 \%$ & 374 & $8.7 \%$ & 243 & $12.2 \%$ & \\
\hline Asian/PI & 65 & $3.2 \%$ & 131 & $3.1 \%$ & 64 & $3.2 \%$ & \\
\hline Other/Unknown & 45 & $2.2 \%$ & 83 & $1.9 \%$ & 28 & $1.4 \%$ & \\
\hline \multicolumn{8}{|l|}{ Comorbidity Score } \\
\hline 0 & 1622 & $79.3 \%$ & 3380 & $78.7 \%$ & 1575 & $79.3 \%$ & \multirow[t]{4}{*}{0.51} \\
\hline 1 & 311 & $15.2 \%$ & 637 & $14.8 \%$ & 301 & $15.1 \%$ & \\
\hline 2 & 76 & $3.7 \%$ & 201 & $4.7 \%$ & 73 & $3.7 \%$ & \\
\hline $3+$ & 36 & $1.8 \%$ & 76 & $1.8 \%$ & 38 & $1.9 \%$ & \\
\hline \multicolumn{8}{|l|}{ Insurance Status } \\
\hline Private Insurance & 881 & $43.1 \%$ & 1410 & $32.8 \%$ & 578 & $29.1 \%$ & \multirow[t]{3}{*}{$<0.001$} \\
\hline Government & 1109 & $54.2 \%$ & 2705 & $63.0 \%$ & 1324 & $66.6 \%$ & \\
\hline Other/Unknown & 55 & $2.7 \%$ & 179 & $4.2 \%$ & 85 & $4.3 \%$ & \\
\hline \multicolumn{8}{|l|}{ Hospital Category } \\
\hline Community & 104 & $5.1 \%$ & 285 & $6.6 \%$ & 126 & $6.3 \%$ & \multirow[t]{5}{*}{$<0.001$} \\
\hline Comprehensive Community & 812 & $39.7 \%$ & 1661 & $38.7 \%$ & 709 & $35.7 \%$ & \\
\hline Academic & 683 & $33.4 \%$ & 1621 & $37.8 \%$ & 815 & $41.0 \%$ & \\
\hline Integrated Network Cancer Program & 404 & $19.8 \%$ & 691 & $16.1 \%$ & 326 & $16.4 \%$ & \\
\hline Other/Unknown & 42 & $2.1 \%$ & 36 & $0.8 \%$ & 11 & $0.6 \%$ & \\
\hline \multicolumn{8}{|l|}{ Clinical Stage } \\
\hline \multicolumn{8}{|l|}{ Overall } \\
\hline 1 & 957 & $46.8 \%$ & 1002 & $23.3 \%$ & 367 & $18.5 \%$ & \multirow[t]{4}{*}{$<0.001$} \\
\hline 2 & 815 & $39.9 \%$ & 2434 & $56.7 \%$ & 1130 & $56.9 \%$ & \\
\hline 3 & 149 & $7.3 \%$ & 657 & $15.3 \%$ & 409 & $20.6 \%$ & \\
\hline Unknown & 124 & $6.1 \%$ & 201 & $4.7 \%$ & 81 & $4.1 \%$ & \\
\hline \multicolumn{8}{|l|}{ Tumor Stage } \\
\hline 1 & 1031 & $50.4 \%$ & 1142 & $26.6 \%$ & 406 & $20.4 \%$ & \multirow[t]{5}{*}{$<0.001$} \\
\hline 2 & 699 & $34.2 \%$ & 2,043 & $47.6 \%$ & 917 & $46.1 \%$ & \\
\hline 3 & 142 & $6.9 \%$ & 598 & $13.9 \%$ & 371 & $18.7 \%$ & \\
\hline 4 & 71 & $3.5 \%$ & 395 & $9.2 \%$ & 246 & $12.4 \%$ & \\
\hline Unknown & 102 & $5.0 \%$ & 116 & $2.7 \%$ & 47 & $2.4 \%$ & \\
\hline \multicolumn{8}{|l|}{ Nodal Stage } \\
\hline 0 & 1667 & $81.5 \%$ & 3234 & $75.3 \%$ & 1431 & $72.0 \%$ & $<0.001$ \\
\hline 1 & 254 & $12.4 \%$ & 757 & $17.6 \%$ & 406 & $20.4 \%$ & \\
\hline $2 / 3$ & 53 & $2.6 \%$ & 151 & $3.5 \%$ & 89 & $4.5 \%$ & \\
\hline Unknown & 71 & $3.5 \%$ & 152 & $3.5 \%$ & 61 & $3.1 \%$ & \\
\hline Histology & & & & & & & \\
\hline Ductal & 1479 & $72.3 \%$ & 2946 & $68.6 \%$ & 1333 & $67.1 \%$ & 0.001 \\
\hline Lobular & 361 & $17.7 \%$ & 916 & $21.3 \%$ & 447 & $22.5 \%$ & \\
\hline Other/Unknown & 205 & $10.0 \%$ & 432 & $10.1 \%$ & 207 & $10.4 \%$ & \\
\hline
\end{tabular}


TABLE 3 continued

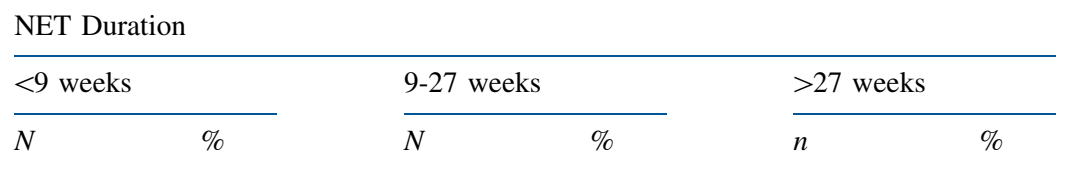

Pathologic Stage

Overall

0/IS

1

2

3

Unknown

Tumor Stage

$$
\text { O/IS }
$$

1

2

3

4

Unknown

Nodal Stage

0

1

2

3

Unknown

Tumor Grade

Well Differentiated

Moderately Differentiated

Poorly/Undifferentiated

Unknown

Surgery

Primary

$$
\text { Lumpectomy }
$$

Mastectomy

Surgery, NOS

Axilla $(* 2012$ and later)

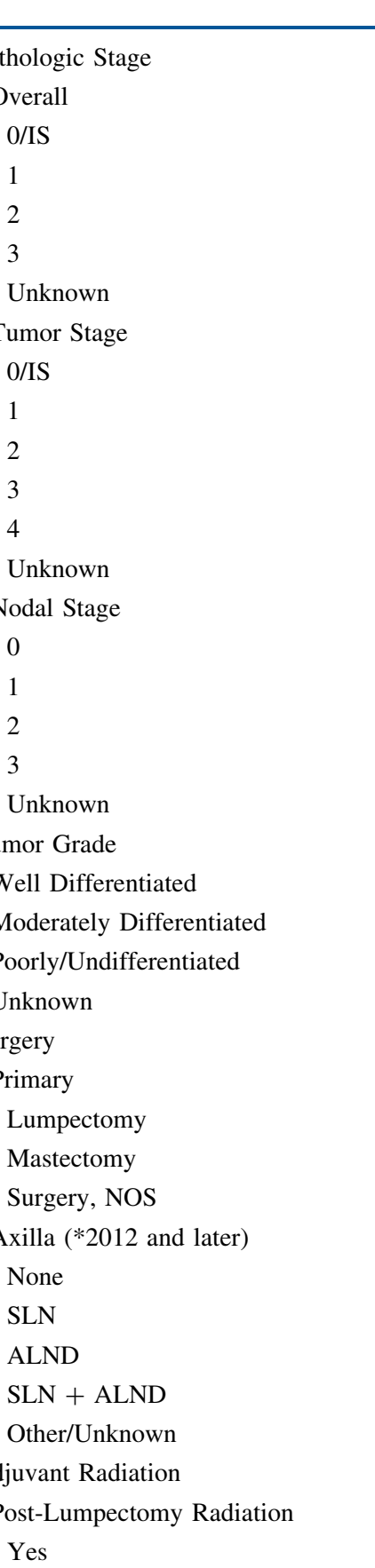

Other/Unknown

Adjuvant Radiation

Post-Lumpectomy Radiation

$\begin{array}{ll}16 & 0.8 \% \\ 790 & 38.6 \% \\ 751 & 36.7 \% \\ 319 & 15.6 \% \\ 169 & 8.3 \%\end{array}$

$23 \quad 1.1 \%$

$1096 \quad 53.6 \%$

$663 \quad 32.4 \%$

$149 \quad 7.3 \%$

$66 \quad 3.2 \%$

$48 \quad 2.3 \%$

1171

484

153

81

156

612

1057

270

106

929

1115

$45.4 \%$

1

127

887

209

305

517

\section{$54.5 \%$}

$0.0 \%$

$29.9 \%$

$51.7 \%$

$13.2 \%$

$5.2 \%$

Yes

No

Unknown

705

221

3

$6.2 \%$

$43.4 \%$

$10.2 \%$

$14.9 \%$

$25.3 \%$

2267
2024
3

$52.8 \%$

$47.1 \%$

$0.1 \%$

1151

$57.9 \%$

$43.3 \%$

$24.8 \%$

$8.5 \%$

$5.3 \%$

$12.3 \%$

$29.2 \%$

$52.7 \%$

$12.4 \%$

$5.7 \%$

428

1737

$10.0 \%$

$40.5 \%$

$13.8 \%$

$13.7 \%$

588

$22.1 \%$

949

$22.1 \%$

\section{4}

2

$42.0 \%$

$0.1 \%$

$15.1 \%$

$38.0 \%$

$14.2 \%$

$11.9 \%$

$20.7 \%$

411

$70.8 \%$

$28.5 \%$

$23.8 \%$

$0.3 \%$

647

15

$0.7 \%$

$28.7 \%$

$70.9 \%$

754

1256

$37.3 \%$

$62.1 \%$

$0.4 \%$

14

$0.7 \%$

697

445

$60.6 \%$

$38.7 \%$

9

$0.8 \%$

334

$40.0 \%$

$4 \quad 0.4 \%$

497

$59.6 \%$

3

$0.4 \%$

$<0.001$ 
TABLE 3 continued

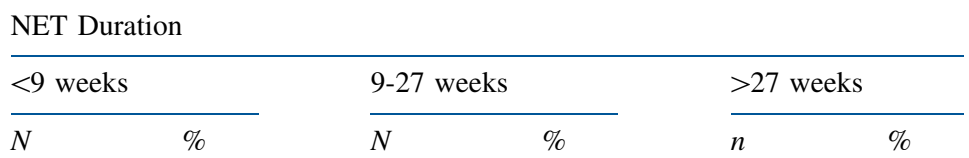

Pathologic Response to NET

Pathologic Tumor Response

Complete

Downstaged

Stable

Upstaged

Unknown

Pathologic Nodal Response

Complete

Downstaged

Stable

Upstaged

Unknown

Overall

Complete

Downstaged

Stable

Upstaged

Unknown

Objective Response

Objective Response ${ }^{\mathrm{a}}$ Rate (ORR)

Upstaged

Unknown

\section{8}

255

1429

200

143

27

9

1276

515

218

0

204

956

530

355

1160

530

355

307

234

40

38 (22-59)

242

\begin{abstract}
$0.9 \%$
$12.5 \%$

$69.9 \%$

$9.8 \%$

$7.0 \%$
\end{abstract}

$1.3 \%$

$0.4 \%$

$62.4 \%$

$25.2 \%$

$10.7 \%$

$0.0 \%$

$10.0 \%$

$46.7 \%$

$25.9 \%$

$17.4 \%$

$56.7 \%$

$25.9 \%$

$17.4 \%$

$<18$

18-30

$>30$

Follow-up (Median (IQR)) (months)

Total Deceased ${ }^{\mathrm{c}}$

${ }^{a}$ Objective response is defined as complete, downstaged, or stable pathologic response.

${ }^{\mathrm{b}}$ Follow-up represents the elapsed time between diagnosis and last contact

${ }^{\mathrm{c}}$ Vital status available for $(6,850,82.3 \%)$ of NET patients

$N E T$ neoadjuvant endocrine therapy, $S D$ standard deviation, $P I$ Pacific Islander, $S L N$ sentinel lymph node biopsy, $A L N D$ axillary lymph node dissection, $I Q R$ interquartile range, $N O S$ not otherwise specified, $N A$ not applicable

There were several other notable findings in this study. First, the unadjusted death rate was significantly higher in patients in the NET group (13.8\%) versus the surgery-first group (7.2\%). This is likely due to the inherent differences in patient characteristics in these two groups. Patients in the NET group were older at time of diagnosis, with higher Charleson-Deyo comorbidity scores, more advanced tumor stage, and less likely to receive adjuvant radiotherapy. This higher unadjusted death rate is not expected in patients treated with NET during the pandemic since these patients are more likely to present with earlier-stage disease and to receive standard adjuvant therapy after their delayed surgery. The possible adverse effects due to surgery delay of cancer patients during the pandemic will be examined using data collected by regional and national medical/surgical organizations (Rachel Jankowitz, MD, personal communication, September 2020).

Second, patients with invasive lobular carcinoma (ILC) were more likely to have disease upstaged after NET. This is unexpected given the higher rate of hormone receptor 


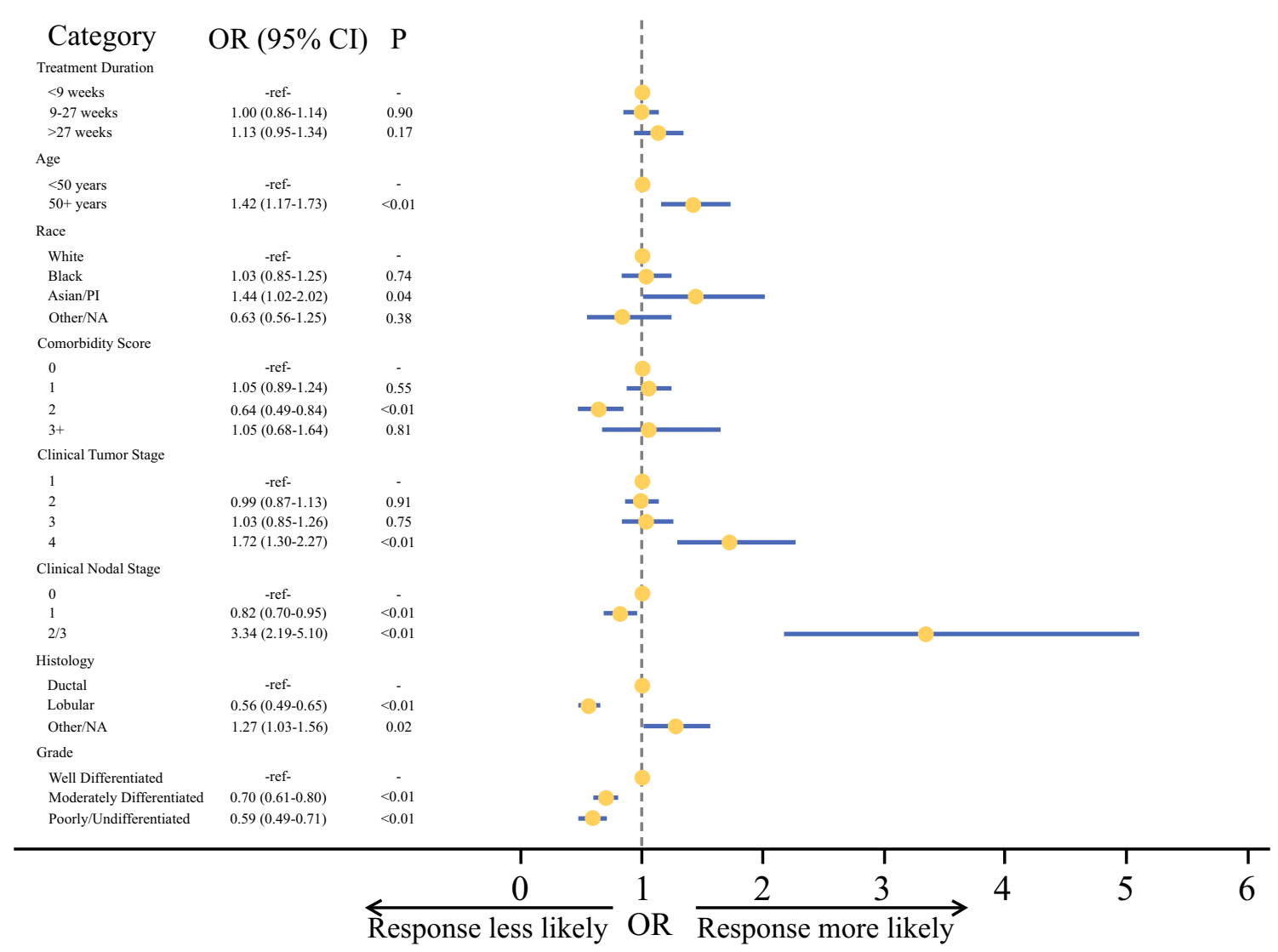

FIG. 3 Forest plot displaying the odds ratio (OR) of response to NET on multivariable logistic regression of entire study cohort $(n=8326)$

positivity (>95\%) in this histologic subtype. ${ }^{15}$ A likely explanation is that clinical stage of ILC may often underestimate the pathologic stage. Specifically, clinical breast examination and breast imaging studies including mammography and ultrasonography often underestimate ILC disease extent. ${ }^{16,17}$ Histologically, ILC tends to be diffusely infiltrating, with invasion of stroma by neoplastic cells in single-cell fashion and often with minimal desmoplastic reaction contributing to the lack of discrete clinical breast examination findings. ${ }^{16}$ The reported sensitivity of mammogram for detecting ILC is only 57-79\%, with up to $30 \%$ not visualized at all. ${ }^{17}$ As the treatment response of ILC is often underestimated by conventional methods, other methods, to accurately assess clinical response, such as measuring the change in \% nuclear positivity for Ki67 using immunohistochemistry, ${ }^{18,19}$ may be more appropriate. However, these data are not available in the NCDB. The effect of NET on clinical outcomes in ILC will await results from an ongoing multicenter study (NCT02206984). ${ }^{20}$

Third, our study showed that Black women were less likely to receive NET compared with their white counterparts. These results highlight the need for health care providers to remain mindful and vigilant when implementing guidelines in managing surgical delays through the COVID-19 pandemic, especially to avoid inadvertently exacerbating racial disparities in breast cancer care as described in a recent editorial. ${ }^{21}$ While we were somewhat reassured by the findings that there were no significant differences in response to NET between white and Black patients in our study, the need to assess NET adherence in these vulnerable populations is warranted, as Black women are reported to have lower rates of endocrine therapy initiation and adherence compared with other racial groups. ${ }^{22}$ Periodic reassessment, as recommended by national medical and surgical organizations, ${ }^{7,9}$ may help to identify those with early disease progression and to direct these patients to more prompt surgical intervention.

A strength of this study is that it is one of the largest studies to evaluate the objective response to NET according to NET duration. Use of NCDB data also highlights real-world practice patterns of NET use, demonstrating a broader range of NET durations than evaluated in previous studies. Weaknesses of this study, inherent to the retrospective study design, include missing data, imbalances of clinical characteristics between subgroups due to selection bias, the inability to ascertain toxicity, compliance, and effectiveness of specific endocrine therapy regimens, and the lack of disease-specific outcome data. Results from this 
study, however, were reassuring and support the use of NET to bridge surgery delay during the COVID-19 pandemic.

In summary, short NET duration $(<9$ weeks $)$ did not result in an inferior objective response. Future study to evaluate the interaction between surgery delay and NET use on clinical outcome will provide insights in the safety of NET use to bridge potential surgery delay in patients with HR+ breast cancer.

\section{DECLARATIONS}

DISCLOSURE Authors have no financial relationships or conflicts of interest relevant to this article to disclose.

\section{REFERENCES}

1. Kohler BA, Sherman RL, Howlader N, et al. Annual report to the nation on the status of cancer, 1975-2011, featuring incidence of breast cancer subtypes by race/ethnicity, poverty, and state. J Natl Cancer Inst. 2015. https://doi.org/10.1093/jnci/djv048.

2. Chiba A, Hoskin TL, Heins CN, et al. Trends in neoadjuvant endocrine therapy use and impact on rates of breast conservation in hormone receptor-positive breast cancer: a National Cancer Data Base Study. Ann Surg Oncol. 2017;24(2):418-24. https://d oi.org/10.1245/s10434-016-5585-5.

3. Dietz JR, Moran MS, Isakoff SJ, et al. Recommendations for prioritization, treatment, and triage of breast cancer patients during the COVID-19 pandemic. The COVID-19 pandemic breast cancer consortium. Breast Cancer Res Treat. 2020;181(3):487-97.

4. Freedman RA, Sedrak MS, Bellon JR, et al. Weathering the storm: managing older adults with breast cancer amid COVID-19 and beyond. J Natl Cancer Inst. 2020. https://doi.org/10.1093/jnc i/djaa079.

5. Marti C, Sánchez-Méndez JI. Neoadjuvant endocrine therapy for luminal breast cancer treatment: a first-choice alternative in times of crisis, such as the COVID-19 pandemic. Ecancermedicalscience. 2020. https://doi.org/10.3332/ecancer.2020.1027.

6. Dowsett M, Ellis MJ, Dixon JM, et al. Evidence-based guidelines for managing patients with primary ER+ HER2 - breast cancer deferred from surgery due to the COVID-19 pandemic. NPJ Breast Cancer. 2020. https://doi.org/10.1038/s41523-020-0168-9.

7. American Society of Breast Surgeons. ASBrS resource guide to endocrine therapy in the COVID-19 pandemic. 2020.

8. Thompson CK, Lee MK, Baker JL, Attai DJ, DiNome ML. Taking a second look at neoadjuvant endocrine therapy for the treatment of early stage estrogen receptor positive breast cancer during the COVID-19 outbreak. Ann Surg. 2020;272(2):e96-7. https://doi.org/10.1097/SLA.0000000000004027.

9. Society for Surgical Oncology. Resource for management options of breast cancer during COVID-19. 2020.
10. Spring LM, Gupta A, Reynolds KL, et al. Neoadjuvant endocrine therapy for estrogen receptor-positive breast cancer a systematic review and meta-Analysis. JAMA Oncol. 2016;2(11):1477-86. https://doi.org/10.1001/jamaoncol.2016.1897.

11. Carpenter R, Doughty JC, Cordiner C, et al. Optimum duration of neoadjuvant letrozole to permit breast conserving surgery. Breast Cancer Res Treat. 2014;144(3):569-76. https://doi.org/10.1007/ s10549-014-2835-8.

12. Bleicher RJ, Ruth K, Sigurdson ER, et al. Time to surgery and breast cancer survival in the United States. JAMA Oncol. 2016;2(3):330-9. https://doi.org/10.1001/jamaoncol.2015.4508.

13. Mallin K, Browner A, Palis B, et al. Incident cases captured in the national cancer database compared with those in U.S. population based central cancer registries in 2012-2014. Ann Surg Oncol. 2019;26(6):1604-12. https://doi.org/10.1245/s10434-019-072131.

14. Leal F, Liutti VT, Antunes dos Santos VC, et al. Neoadjuvant endocrine therapy for resectable breast cancer: a systematic review and meta-analysis. Breast. 2015;24(4):406-12.

15. Christgen M, Steinemann D, Kühnle E, et al. Lobular breast cancer: clinical, molecular and morphological characteristics. Pathol Res Pract. 2016;212(7):583-97. https://doi.org/10.1016/j. prp.2016.05.002.

16. Johnson K, Sarma D, Hwang ES. Lobular breast cancer series: imaging. Breast Cancer Res. 2015. https://doi.org/10.1186/s130 58-015-0605-0.

17. Porter AJ, Evans EB, Foxcroft LM, Simpson PT, Lakhani SR. Mammographic and ultrasound features of invasive lobular carcinoma of the breast. J Med Imaging and Radiat Oncol. 2014;58(1):1-10. https://doi.org/10.1111/1754-9485.12080.

18. Dowsett M, Smith IE, Ebbs SR, et al. Prognostic value of Ki67 expression after short-term presurgical endocrine therapy for primary breast cancer. J Natl Cancer Inst. 2007;99(2):167-70. https://doi.org/10.1093/jnci/djk020.

19. Suman VJ, Ellis MJ, Ma CX. The ALTERNATE trial: assessing a biomarker driven strategy for the treatment of post-menopausal women with Er+/Her2- invasive breast cancer. Chin Clin Oncol. 2015. https://doi.org/10.3978/j.issn.2304-3865.2015.09.01.

20. Endocrine response in women with invasive lobular breast cancer. ClinicalTrials.gov identifier: NCT02206984. https://clinica ltrials.gov/ct2/show/NCT02206984. 2020.

21. Obeng-Gyasi S, Oppong B, Paskett ED, Lustberg M. Purposeful surgical delay and the coronavirus pandemic: how will black breast cancer patients fare? Breast Cancer Res Treat. 2020. https://doi.org/10.1007/s10549-020-05740-0.

22. Roberts MC, Wheeler SB, Reeder-Hayes K. Racial/ethnic and socioeconomic disparities in endocrine therapy adherence in breast cancer: a systematic review. Am J Public Health. 2015;105:e4-15. https://doi.org/10.2105/AJPH.2014.302490.

Publisher's Note Springer Nature remains neutral with regard to jurisdictional claims in published maps and institutional affiliations. 